\title{
Introduction : \\ Monde animal et végétal dans le récit bref du Moyen Âge
}

\author{
Hugo O. Bizzarri (Fribourg)
}

L'idée inspiratrice de ce volume provient d'une phrase de Robert Delort, dans son livre consacré au tissage des lignes générales de ce qu'il a alors appelé la 〈zoohistoire 〉: «il n'est pas possible de dissocier entièrement l'histoire des animaux de celle des hommes ». ${ }^{2}$ L'homme a maintenu une relation ancestrale avec les animaux qui s'est redéfinie d'époque en époque. Il en va de même de l'entourage dans lequel lui et les animaux ont évolué, ce que l'on a pour habitude de désigner sous l'enseigne d'environnement. C'est pour cela que notre perspective ne s'arrête pas à l'observation des relations que l'homme a maintenues uniquement avec les animaux, mais elle essaie également d'englober et de ne pas laisser en marge le contexte géographique et vital dans lequel les deux ont évolué. Nous pourrions dire qu'il s'agit d'une relation triangulaire dans laquelle chaque élément a joué un rôle fondamental et dont l'homme est un angle complementaire.

Si les relations de l'être humain avec son entourage ont varié au fil du temps, il en va de même pour ses formes de représentation et la signification qu'il a donnée à chaque élément. Le regard de l'homme médiéval sur son entourage est un regard plein de significations. Il suffit de feuilleter la grande encyclopédie d'Alexandre Neckam, < De naturis rerum ', pour percevoir une multiplicité de résonances transcendantes que l'univers lui évoquait. Pour l'encyclopédiste, chaque élément de la nature contient dans son essence l'empreinte de Dieu, et ainsi la connaissance du monde se transforme en tâche transcendante. ${ }^{3}$ Pour cette raison, les aspects primordiaux de la Création (les anges, le firmament, le temps, les étoiles, les planètes) sont interprétés à partir des passages initiaux de la Genèse. Cette vision du monde fondée sur les valeurs transcendantes de la Bible est celle

I PL 2 I0, col. 579.

2 Delort, Robert, Les animaux ont une histoire (Points. Histoire I74), Paris I984, p. Io I.

3 Decrevit itaque parvitas mea quarundam rerum naturas scrito commendare, ut proprietatibus ipsarum investigatis ad originem ipsarum, ad rerum vidilicet opificem, mens lectoris recurrat, ut ipsum admirans in se et in creaturis suis pedes Creatoris, justitiam scilicet et misericordiam, spiritualiter osculetur. Alexander Neckam, De naturis rerum, éd. par Wright, Thomas, Nendeln 1967, p. 2. 
qu'offre Gonzalo de Berceo dans l'Introduction à sa collection de < Milagros > mariaux. Le pré dans lequel arrive le poète est le reflet du jardin d'Eden (Genèse $2: 8$ ) qui, après être décrit, nécessite d'être interprété. Alors seulement Berceo dévoile qu'il s'est exprimé avec un langage caché [palabra escura] (c. I6b). Le fameux vers tolgamos la cortesa, al meollo entremos (enlevons l'écorce, allons au centre) (c. I6c) n'est rien d'autre qu'une invitation à jeter un regard plus profond sur le monde et découvrir les résonnances bibliques qui, comme en filigrane, se retrouvent cachées dans chaque élément de l'univers.

Mais l'analyse d'Alexander Neckam sur la nature des choses n'est pas une analyse partielle; bien au contraire, nous pourrions la considérer comme globale. S’il parle des poissons, il évoque avant les mers et les fleuves ; s'il parle des oiseaux, il mentionne auparavant l'air ; s'il parle de la terre, il en vient ensuite à la végétation et aux animaux. Alexander Neckam ne dissocie pas le milieu de ses habitants.

Dans le travail < scientifique > des encyclopédistes, le point important n'était pas tant la description objective du phénomène, mais surtout ses implications symboliques et son rapport à l'homme. Si le lion - et précédemment l'ours - ont été vus comme rois des quadrupèdes et si l'aigle était le roi des oiseaux, ${ }^{4}$ le monde marin ne connaîssait pas d'espèce dominante et était vu comme une représentation de la société idéale. Ainsi Pierre Damien, dans sa lettre < De bono religiosi status et variorum animalium >, comparait la congrégation des moines du monastère avec un banc de poissons :

Claustrum quippe monasterium vivarium est animarum. Ibi quippe vivunt pisces, que juxta legis edicta pennulas habent atque ut in corpus Christi transformantur, Israelitarum mensis delicias praebent (col. 766).

Le cloître pour Damien est un monde en harmonie dans lequel il n'y a pas de différences sociales, une société, en définitive, parfaite.

Cependant, l'homme médiéval n'a pas toujours donné une interprétation univoque de l'environnement. Le Moyen Âge est une époque dans laquelle le discours scientifique et les croyances populaires s'entrecroisent. Le proverbe minores pisces a majoribus devorari (le grand poisson mange le plus petit), ${ }^{6}$ qui a eu autant d'impact sur Brueghel

4 Cette question a été posée par Michel Pastoureau dans plusieurs de ses travaux. Pastoureau, Michel, Quel est le roi des animaux ?, dans : Le monde animal et ses répresentations au moyen âge $\left(\mathrm{XI}^{\mathrm{e}}-\mathrm{XV}^{\mathrm{e}}\right.$ siècles), actes du $\mathrm{XV}^{\text {ème }}$ Congrès de la Société des Historiens Médiévistes de l'Enseignement Supérieur Public, Toulouse, 25-26 mai 1984, Toulouse I985, pp. I33-I42 ; id., Comment le bestiaire médiéval s'est donné un roi, dans : Une histoire symbolique du Moyen Âge occidental, Paris 2004, pp. 54-72 et id., L'ours. Histoire d'un roi déchu (Points H472), Paris 2007.

$5 \quad$ PL i45, col. $763-792$.

6 Il apparaît pour la première fois chez Varon, < Menippeae > et ensuite chez Saint Augustin, <Enarrationes in Psalmos >. Voir Singer, Samuel, Thesaurus proverbiorum Medii Aevi. Lexikon der Sprichwörter des romanisch-germanischen Mittelalters, Berlin/New York 1996, vol 3, pp. 263-264; Schenda, Rudolf, Fisch, Fischen, Fischer, dans : Enzyklopädie des Märchens, éd. par Ranke, Kurt, Berlin/New York 1984, vol. 4, col. I I96-I 2 I I ; Bambeck, Manfred, < Die großen Fische fressen die kleinen >. Bemerkungen zu einem patristischen Traditionshintergrund für Hyeronimus Bosch und Pieter Bruegel, dans : Neuphilologische Mitteilungen 82 (I98I), pp. 262-268. Celle de Latini est sa seule documentation en Espagne 
et qui offrait, d'après Rudolf Schenda, une < métaphore du monde > (Metaphern von der Welt), a inspiré à Brunetto Latini une interprétation différente du monde marin. ${ }^{7}$ En parlant des poissons, il signale :

Dont li i. manguënt herbes et petites vermines, et li autre menguënt autres poissons; et ce est par une tele maniere ke toujours li moindres est viande del grignour, et ensi vit li uns de l'autre. ${ }^{8}$

Latini pourrait s'être arrêté sur la description de cette caractéristique des poissons, mais il ajoute que les puissants soumettent les plus faibles (vit li uns de l'autre), ${ }^{9}$ donnant au passage une signification sociale. De même chez l'auteur anonyme du < Libro de Alexandre , le monde marin est symbole d'oppression sociale. Quand Alexandre le Grand descend dans les profondeurs de l'océan grâce à sa capsule, il observe pendant quinze jours les coutumes des poissons. Il conclut alors :

Otra fazaña vio en estos pobladores :

vio que los mayores comien a los menores;

los chicos a los grandes tenienlos por señores;

maltrayen los mas fuertes a todos los menores.

Diz’ el rey : ¡ Sobervia es en todos lugares ! :

es fuerça en la tierra e dentro en los mares ;

las aves eso mismo non se catan por pares.

¡ Dios confunda tal viçio que tien’ tantos lugares ! ${ }^{\text {10 }}$

Ce qui surprend Alexandre le Grand est le fait de constater que le monde marin est un reflet des autres qu'il connaît : l'humain et l'aérien, et que dans tous l'Orgueil prédomine et fait que le puissant soumette le faible. ${ }^{\text {II }}$

durant le Moyen Âge, mais elle n’a pas été enregistrée par O’Kane, Eleanor S., Refranes y frases proverbiales españolas de la Edad Media, Madrid I959.

7 Sullivan, Margaret A., Bruegel's Proverb Painting : Renaissance Art for a Humanist Audiens, dans : Wise Word. Essays on the Proverb, éd. par Mieder, Wolfgang, London/New York 1994, pp. 253-295; The Nederlandish Proverbs. An International Symposium of the Peter Brueghels, éd. par Mieder, Wolfgang, Burlington 2004.

8 Latini, Brunetto, Li livres dou tresor, éd. par Carmody, Francis J., Los Angeles I948, p. I 27. Je trouve très intéressante la traduction castillane de ce passage, datée de la fin du XIII ${ }^{\mathrm{c}}$ siècle : Ay algunos que comen yerba e gusaniellos pequeños, e otros que comen pescados; e esto es asy que sienpre los mayores comen a los mas pequeños, que son los unos governadores de los otros. Latini, Brunetto, Libro del tesoro. Versión castellana de < Li Livres dou tresor >, éd. par Baldwin, Spurgeon, Madison i 989, p. 69.

9 Dans la traduction castillane : que son los unos governadores de los otros.

Io Libro de Alexandre, éd. par Casas Rigall, Juan, Madrid 2007, c. 2316-2317. Le poète suit ici l'< Alexandreis > (X, I-5), l'< Historia de Preliis $\mathrm{J}^{2}$ > et le < Roman d'Alexandre > (B, v. 856 867 et 7725-7736) ; voir Willis, Raymond, S., The Relationship of the Spanish < Libro de Alexandre > to the < Alexandreis > of Gautier de Châtillon, Princeton/Paris I934, pp. 49-50 et id., The Debt of the Spanish < Libro de Alexandre > to the French < Roman d'Alexandre >, Princeton I935, pp. 3I-39.

I I Pour une vision complète des aspects naturalistes de la légende d'Alexandre, je renvoie à la quatrième partie de l'œuvre collective dirigée par Gaullier-Bougassas, Catherine, La 
L'univers pour l'homme médiéval est rempli de significations qui sont souvent contradictoires. Un même objet de la réalité peut avoir un sens positif ou négatif. C'est ce qu'a fait Eudes de Chériton avec l'interprétation de ses <Fabulae > traduites au castillan en tant que < Libro de los gatos >. Chaque récit réveille chez le prédicateur plus d'une interprétation, parce qu'il considère, bien entendu, que les signes du monde n'ont pas un sens univoque. ${ }^{\mathrm{I2}}$ Cette multiplicité sémantique apparaissait déjà dans le texte biblique présentant l'arbre du bien et du mal et l'arbre du soleil et de la lune. La culture médiévale est aussi la culture de l'arbre. La vedette du paysage est l'arbre. Il se distingue dans la forêt et dans le jardin. ${ }^{\mathrm{I}}$ Les encyclopédies lui consacrent des sections spéciales, le rangeant toujours aux côtés des herbes aromatiques et parfois transformant les herbes en arbres aromatiques. Les vices et vertus étaient représentés sous forme arborescente. On y indiquait la chaîne de ramifications qu'ils possédaient. Le prêtre dominicain Jacopo da Benavente dans son traité < Viridarium > offre les vices représentés sous forme d'arbre enraciné sur le péché d'Orgueil. ${ }^{\mathrm{I}}$ Pendant que l'âme est représentée comme un verger dont les épines sont les péchés et les fleurs les vertus. ${ }^{\text {Is }}$

Juan Manuel dans le <Conde Lucanor> (ex. 26) raconte les évènements de l'arbre du Mensonge et de la Vérité, un récit allégorique qui tente d'expliquer la présence du Mensonge dans la société et pourquoi ceux qui le suivent finissent malheureux. ${ }^{16}$ De son côté, Alexandre le Grand, dans ses infatigables voyages, trouve le Temple de Phébus et Diane (<Libro de Alexandre `, c. 2477-2494) où l'on retrouve l'arbre mythique du soleil et de la lune qui lui annonce sa mort prochaine. Le protagonisme de l'arbre dans la culture médiévale l'a transformé en un élément indépendant du paysage. ${ }^{17}$

fascination pour Alexandre le Grand dans les littératures européenes ( $\mathrm{X}^{\mathrm{e}}-\mathrm{XVI}^{\mathrm{e}}$ siècle), Turnhout 2014, vol. 3, pp. I273-1707.

I 2 Je renvoie à la collaboration de Bernard Darbord dans ce volume pour comprendre ce processus.

I 3 La forêt a été caracterisée comme un <ensemble complexe > dans lequel l'arbre est son composant primordial ; voir Bechmann, Roland, Des arbres et des hommes. La forêt au Moyen Âge, Paris 1984 .

I 4 Il a été traduit au castillan au XIV siècle sous le titre de < Viridario > ou < Vergel de consolaçión del alma > : [...] es sobervia como en cabeça de los otros pecados es a enpeçar, ca de la rayz de esa mesma salen syete pecados mortales. Tractado de viçios e virtudes. An Edition with Introduction and Glossary, éd. par Johnson, Cleveland, Ptomac I993, p. 82.

Is Destas las dos partes primeras que fablan e que se contienen de todos cardos e de espinas que omne debe echar del vergel del alma para la alinpiar e lo ordenar bien por que pueda en el plantar arboles e frutos e yerbas de virtud e flores apuestas e de buen olor. Tractado de vicios e virtudes (note I4), p. I 25 .

I6 Á ce propos, voir les collaborations d'Olivier Biaggini et Natacha Crocoll dans ce volume.

I7 L'arbre a également trouvé sa place dans les visions et descriptions de l'Au-delà ; voir Patch, Howard R., El otro mundo en la literatura medieval (Lengua y estudios literarios), trad. par Hernández Campos, Jorge, México I 983 et Uría Maqua, Isabel, El árbol y su significación en las visiones medievales del otro mundo, dans : Revista de literatura medieval i (1989), pp. $103-122$. 
À plusieurs reprises l'on peut observer que le monde végétal au Moyen Âge est un monde animé, un être vivant. ${ }^{18}$ Michel Pastoureau a attribué cette qualité à la forêt : "Pour la culture médiévale, le bois est d'abord une matière vivante ", ${ }^{19}$ mais cela peut s'appliquer à tout le monde végétal. La ténébrosité de la forêt de Corpes (Robledo de Corpes) dans le < Cantar de Mio Cid >, dont les grands arbres pujan con las nues (luttent avec les nuages) (v. 2698) n'est pas un simple topique littéraire ou paysage épique comme le caractérisait Curtius, mais un complice de l'action des infants. ${ }^{20} \mathrm{Il}$ est ténébreux lorsque les amants ont l'intention trouble des maris de maltraiter leurs femmes, et doux (fallaron un vergel con una linpia fuent', ils trouvèrent un verger avec une fontaine cristalline, v. 2700) quand les maris doivent feindre l'amour. Le paysage change à l'unisson les intentions des infants de Carrión et, tout comme eux, cache sa dangerosité. ${ }^{21}$

À l'opposé de la forêt se trouve le jardin, la végétation domestique et domestiquée, qui pour l'homme était symbole de Paradis. ${ }^{22}$ Le jardin est aussi un être animé. Dans < La Celestina `, avant l'arrivée de Calixte au verger de Mélibée, toute la nature est contaminée par le désir sensuel de la jeune femme qui attend son amant :

Todo se goza este huerto con tu venida. Mira la luna cuan clara se nos muestra. Mira las nubes como huyen. Oye la corriente agua desta fontecica cuanto mas suave murmurio y zurrio lleva por entre las frescas verduras. Escucha los altos cipreses como se dan paz unos ramos con otros por intercesion de un templadico viento que los menea. Mira sus quietas sombras cuan escuras estan y aparejadas para encobrir nuestro deleite. ${ }^{23}$

Il ne s'agit pas seulement d'un lieu agréable, toile de fond d'une scène amoureuse, mais d'un érotisme senti tant par la jeune femme que par le jardin qui sera la scène d'un épisode amoureux. ${ }^{24}$

Je reviens sur l'idée initiale : l'histoire du monde animal et végétal est aussi l'histoire de l'homme, de sa vision du monde et de sa manière de le représenter. Pour l'homme

I 8 Voir l'étude de Rippmann, Dorothee, [...] das die Erde die Mutter und die Sonne der Vater der Pflanzen ist. Bartholomeus Anglicus' enzyklopädisches Wissen über Pflanzen im Solarzeitalter, dans : Mitteilungen des Instituts für österreichische Geschichtsforschung I 23 (2015), pp. 34I-370.

I9 Pastoureau (note 4), pp. 92-95.

20 Curtius, Ernst R., Literatura europea y Edad Media latina (Lengua y Estudios Literarios), trad. Frenk, Margit et Alatorre, Antonio, México I955, vol. I, pp. 286-289. Voir également les commentaires d'Alberto Montaner Frutos dans son édition Cantar de Mio Cid, Barcelona 2007 , pp. 619-620.

2 I La forêt, que Jacques Le Goff a caractérisée comme « équivalent médiéval du désert oriental » est un lieu de solitude, d'épreuves et de dangerosité dans la littérature épique médiévale. Voir Le Goff, Jacques, Le désert-forêt dans l’Occident médiéval, dans : L'imaginaire médiéval (Bibliothèque des Histoires), Paris I985, pp. 59-75.

22 Haudebourg, Marie-Thérèse, Les jardins du moyen âge, Paris 2001.

23 Rojas, Fernando de, La Celestina, éd. par Lobera, Francisco J., Serés, Guillermo, Díaz-Mas, Paloma et alii, Madrid 20I I, pp. 320-32 I.

24 Malheureusement, nous conservons peu de vestiges des jardins médiévaux, remodelés par l'architecture de la Renaissance. Les allusions littéraires se transforment donc en précieux 
médiéval l'univers était rempli de résonances symboliques. Je n'entends pas sousentendre ainsi que pour l'esprit moderne l'entourage lui soit indifférent. Les différents sommets pour résoudre les problèmes climatiques expriment bien cette préoccupation. Mais pour l'homme moderne l'animal et le végétal sont $<$ l'autre $>$ contre lequel l'être humain agit. Pour l'homme médiéval, ils sont partie intégrante de lui-même. Cela explique que Brunetto Latini, dans son < Livres dou tresor >, fasse précéder la connaissance de l'être humain par l'histoire, la géographie, les plantes et les animaux, sans lesquels l'on ne comprendrait pas la nature humaine. ${ }^{25}$

Le présent volume est une tentative d'approfondir ces multiples significations et représentations que l'homme médiéval a eu du monde animal et végétal. Seize experts en la matière se sont donné rendez-vous pour réaliser deux journées d'échange dans les Universités de Fribourg et Genève. La souris, l'éléphant, le singe, les oiseaux, la forêt, les proverbes animalistiques, les destins croisés de la nature, sont quelques-uns des thèmes qui ont été soumis à réflexion. Tous témoignent de l'entremêlement indissociable des êtres et des espaces dans l'imaginaire médiéval.

Nota bene : Le colloque <Monde animal et végétal dans le récit bref du Moyen Âge > a eu lieu les 2 et 3 mai 2016 aux Universités de Fribourg et Genève. Il a reçu le soutien des universités partenaires, du Fonds National Suisse et de l'Académie de Sciences Humaines et Sociales. L'éditeur remercie l'accueil généreux que l'Institut des Études Médiévales de l'Université de Fribourg a donné à ce volume. Une mention spéciale mérite le Prof. Carlos Alvar qui nous a acqueilli à Genève et Adrián Fernández et Martin Rohde qui ont énormément contribué à la préparation de ce livre.

témoignages pour leur connaissance, même si souvent lesdites allusions sont plus superficielles que nous le souhaitons. Je renvoie au volume collectif Jardins et vergers en Europe occidentale (VIII'-XVIII ' siècles), Auch I989, avec d'importantes contributions de Philippe Ménard sur le jardin dans le < roman courtois >, Lucie Bolens sur le jardin andalous et Henri Bresc sur le jardin sicilien, entre autres.

25 Et porce dit le maistres que la premiere partie de son tresor est en derniers contanz. Car si comme les genz ne porroient nie chevir lor besoignes se lor marcheandies sans monoie, tout autresi se porroient il savoir la certenité des bumaines choses, se il ne seussent ce que ceste premiere partie raconte, Latini, Brunetto, Li livres dou tresor (note 8), p. I7I. 